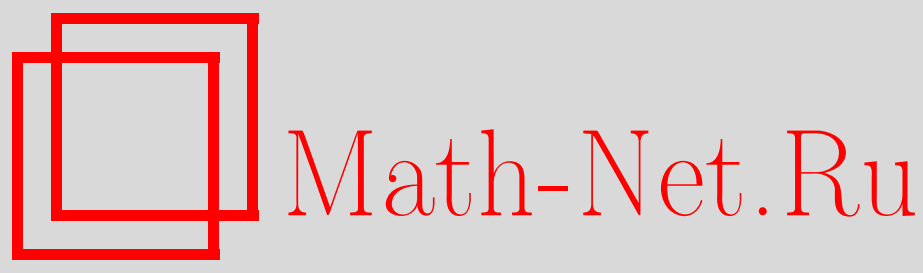

Ю. Х. Эшкабилов, О спектре одного модельного трехчастичного оператора Шредингера, ТМФ, 2016, том 186, номер 2, 311-322

DOI: https://doi.org/10.4213/tmf8879

Использование Общероссийского математического портала Math-Net.Ru подразумевает, что вы прочитали и согласны с пользовательским соглашением http://www . mathnet.ru/rus/agreement

Параметры загрузки:

IP : 3.91 .87 .62

26 апреля 2023 г., 13:49:59

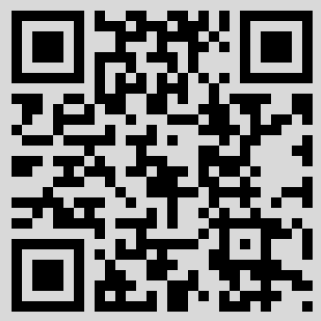




\section{О СПЕКТРЕ ОДНОГО МОДЕЛЬНОГО ТРЕХЧАСТИЧНОГО ОПЕРАТОРА ШРЕДИНГЕРА}

Изучается спектр некоторого модельного трехчастичного оператора Шредингера $H(\varepsilon), \varepsilon>0$. Доказано, что при достаточно малом $\varepsilon>0$ у этого оператора отсутствуют связанные состояния и двухчастичные ветви спектра. Получена оценка для малого параметра $\varepsilon$.

Ключевые слова: операторы Шредингера, спектр, существенный спектр, дискретный спектр.

DOI: $10.4213 / \operatorname{tmf} 8879$

\section{1. ВВЕДЕНИЕ}

Пусть $\Omega_{1}=[a, b]^{\nu_{1}} \subset \mathbb{R}^{\nu_{1}}$ и $\Omega_{2}=[c, d]^{\nu_{2}} \subset \mathbb{R}^{\nu_{2}}$ (где $\nu_{1}, \nu_{2} \in \mathbb{N}$ ). Пусть $k_{1}(x, s, y)$ и $k_{2}(x, t, y)$ - непрерывные функции на $\Omega_{1}^{2} \times \Omega_{2}$ и на $\Omega_{1} \times \Omega_{2}^{2}$ соответственно, причем $k_{1}(x, s, y)=\overline{k_{1}(s, x, y)}, k_{2}(x, t, y)=\overline{k_{2}(x, y, t)}$.

В гильбертовом пространстве $L_{2}\left(\Omega_{1} \times \Omega_{2}\right)$ определим линейные ограниченные операторы $T_{1}$ и $T_{2}$ формулами

$$
\begin{aligned}
& T_{1} f(x, y)=\int_{\Omega_{1}} k_{1}(x, s, y) f(s, y) d \mu_{1}(s), \\
& T_{2} f(x, y)=\int_{\Omega_{2}} k_{2}(x, t, y) f(x, t) d \mu_{2}(t),
\end{aligned}
$$

где $\mu_{j}$ - мера Лебега на $\sigma$-алгебре измеримых по Лебегу подмножеств множества $\Omega_{j}, j=1,2$, т. е. здесь интеграл понимается в смысле Лебега. Операторы $T_{1}$ и $T_{2}$ называются частично интегральными операторами (ЧИО) в $L_{2}\left(\Omega_{1} \times \Omega_{2}\right)$.

Пусть $k_{0}(x, y)$ - вещественнозначная непрерывная функция на $\Omega_{1} \times \Omega_{2}$ и $k(x, y ; s, t)$ непрерывная функция на $\left(\Omega_{1} \times \Omega_{2}\right)^{2}$, причем $k(x, y ; s, t)=\overline{k(s, t ; x, y)}$. Обозначим через $H_{0}$ оператор умножения на функцию $k_{0}(x, y)$ :

$$
H_{0} f(x, y)=k_{0}(x, y) f(x, y), \quad f \in L_{2}\left(\Omega_{1} \times \Omega_{2}\right),
$$

* Механико-математический факультет, Национальный университет Узбекистана им. М. Улугбека, Ташкент, Узбекистан. E-mail: yusup62@mail.ru 
а через $K$ обозначим компактный интегральный оператор, заданный на $L_{2}\left(\Omega_{1} \times \Omega_{2}\right)$ равенством

$$
K f(x, y)=\int_{\Omega_{1}} \int_{\Omega_{2}} k(x, y ; s, t) f(s, t) d s d t .
$$

Здесь и в дальнейшем $d s=d \mu_{1}(s), d t=d \mu_{2}(t)$, и мы предполагаем, что меры $\mu_{1}(\cdot)$ и $\mu_{2}(\cdot)$ нормированы, т. е. $\mu_{1}\left(\Omega_{1}\right)=\mu_{2}\left(\Omega_{2}\right)=1$.

Для каждого $\varepsilon>0$ рассмотрим линейный ограниченный оператор

$$
H=H(\varepsilon): L_{2}\left(\Omega_{1} \times \Omega_{2}\right) \rightarrow L_{2}\left(\Omega_{1} \times \Omega_{2}\right),
$$

заданный равенством

$$
H(\varepsilon)=H_{0}-\varepsilon\left(T_{1}+T_{2}\right)-\varepsilon K .
$$

Оператор $H$ является самосопряженным на $L_{2}\left(\Omega_{1} \times \Omega_{2}\right)$. Операторы такого вида возникают, например, при изучении двухчастичных ветвей спектра гамильтонианов, описывающих квантовые системы с большим (или бесконечным) числом частиц [1]-[3], в генераторах сложных марковских процессов, в теории упругости, гидродинамике и других разделах механики [4], [5], а также в теории дискретных трехчастичных операторов Шредингера [6]-[10].

В работе [6] была исследована структура спектра гамильтониана $H^{\mathrm{t}}$ (оператора триплета) системы, состоящей из двух свободно движущихся электронов (из двух электронов с разным спином) и одной примеси на решетке. Гамильтониан $H^{\mathrm{t}}$ имел вид $H^{\mathrm{t}}=H_{0}-\left(T_{1}+T_{2}\right)$, где оператор $H_{0}$ соответствует свободному движению двух электронов и одной примеси (т.е. $H_{0}$ - оператор кинетической энергии), интегральный оператор $T_{1}$ описывает взаимодействие первого электрона с примесью, а интегральный оператор $T_{2}$ - взаимодействие второго электрона с примесью, т. е. оператор $T=T_{1}+T_{2}$ задает потенциал. Гамильтониан $H^{\mathrm{t}}$ в работе [6] был назван трехчастичным оператором триплета (два электрона и одна примесь). В указанной работе изучалась общая модель

$$
\widehat{H}=H_{0}-\left(T_{1}+T_{2}\right)
$$

трехчастичного дискретного оператора $H^{\mathrm{t}}$ и была доказана теорема о существенном спектре гамильтониана $\widehat{H}$.

Дискретный спектр гамильтониана $\widehat{H}$ для произвольных непрерывных ядер изучался в статье [11]. Конечность и бесконечность дискретных спектров трехчастичных операторов, описывающихся моделью $\widehat{H}(1.2)$, были исследованы в рабо$\operatorname{Tax}[7]-[10]$.

Спектральные свойства гамильтониана $H(\varepsilon)(1.2)$ при достаточно малом $\varepsilon>0$ исследованы в работе Минлоса и Акчурина [12], где изучалась теория рассеяния для оператора $H$. Пусть $\nu_{1}=\nu_{2}=d \geqslant 3$ и $k_{0}(x, y)=\omega(x)+\omega(y)$, где $\omega$ - вещественнозначная бесконечно дифференцируемая функция, имеющая единственную критическую точку $q_{0}$, являющуюся точкой невырожденного минимума, а я́дра $k_{1}$, $k_{2}, k$ - гладкие функции. При этих предположениях доказано что при достаточно малом $\varepsilon$ спектр гамильтониана $H$ совпадает со спектром мультипликатора $H_{0}$, т. е. $\sigma(H)=\sigma\left(H_{0}\right)$, следовательно, отсутствуют двухчастичные ветви спектра гамильтониана $H$ и связанные состояния. 
В настоящей работе исследуется спектр гамильтониана $H(\varepsilon)(1.1)$ при достаточно малом $\varepsilon>0$ для произвольных непрерывных функций $k_{0}(x, y), k_{1}(x, s, y)$ и $k_{2}(x, t, y)$ в случае $K=\theta$, где $\theta$ - нулевой оператор. Таким образом, интегральный оператор $K$ отсутствует в модели $H(\varepsilon)(1.1)$. Следовательно, гамильтониан $H(\varepsilon)$ соответствует трехчастичному оператору $\widehat{H}(1.2)$. Получена оценка (2.6) для значений параметра $\varepsilon>0$, при которых спектр гамильтониана $H(\varepsilon)$ совпадает со спектром мультипликатора $H_{0}$ (теорема 2.3), и тем самым отсутствуют двухчастичные ветви спектра трехчастичного оператора $H$ и связанные состояния. Эта оценка важна для проведения экспериментальных исследований и изучения энергии трехчастичных гамильтонианов.

\section{2. НЕКОТОРЫЕ ОБОЗНАЧЕНИЯ И НЕОБХОДИМЫЕ СВЕДЕНИЯ. ФОРМУЛИРОВКА ОСНОВНЫХ ТЕОРЕМ}

Определим операторы $H_{k}(\varepsilon)=H_{0}-\varepsilon T_{k}, k=1,2$. Пусть $R_{\lambda}\left(H_{0}\right)$ - резольвента мультипликатора $H_{0}$,

$$
R_{\lambda}\left(H_{0}\right)=\left(H_{0}-\lambda E\right)^{-1}, \quad \lambda \in \rho\left(H_{0}\right),
$$

где $E$ - тождественный оператор, $\rho\left(H_{0}\right)$ - множество регулярных точек оператора $H_{0}$. Расмотрим ЧИО

$$
W_{k}(\lambda)=T_{k} R_{\lambda}\left(H_{0}\right), \quad \lambda \in \rho\left(H_{0}\right), \quad k=1,2 .
$$

Обозначим через $D_{1}(y ; z, \varepsilon)$ и $D_{2}(x ; z, \varepsilon)$ детерминанты ЧИО $E-\varepsilon W_{1}(z)$ и $E-\varepsilon W_{2}(z)$, где $z \in \rho\left(H_{0}\right)$ [13], [14]. Пусть множества $\sigma_{k}(\varepsilon) \subset \mathbb{C}, k=1,2$, заданы равенствами

$$
\begin{aligned}
& \sigma_{1}(\varepsilon)=\left\{\lambda \in \rho\left(H_{0}\right): D_{1}\left(y_{0} ; \lambda, \varepsilon\right)=0 \text { для некоторого } y_{0} \in \Omega_{2}\right\} \\
& \sigma_{2}(\varepsilon)=\left\{\lambda \in \rho\left(H_{0}\right): D_{2}\left(x_{0} ; \lambda, \varepsilon\right)=0 \text { для некоторого } x_{0} \in \Omega_{1}\right\} .
\end{aligned}
$$

Тогда в силу теоремы 6 из работы [15]

$$
\sigma\left(H_{k}(\varepsilon)\right)=\sigma\left(H_{0}\right) \cup \sigma_{k}(\varepsilon), \quad k=1,2 .
$$

Согласно теореме 3.3 из работы [4] о существенном спектре ЧИО для трехчастичного оператора $H=H(\varepsilon)=H_{0}-\varepsilon\left(T_{1}+T_{2}\right)$ имеем

$$
\sigma_{\text {ess }}(H(\varepsilon))=\sigma\left(H_{0}\right) \cup \sigma\left(H_{1}(\varepsilon)\right) \cup \sigma\left(H_{2}(\varepsilon)\right)=\sigma_{0} \cup \sigma_{1}(\varepsilon) \cup \sigma_{2}(\varepsilon),
$$

где $\sigma_{0}=\sigma\left(H_{0}\right)$, а $\sigma\left(H_{1}(\varepsilon)\right), \sigma\left(H_{2}(\varepsilon)\right)$ соответствуют двухчастичной ветви.

Пусть $\lambda \in \rho\left(H_{0}\right)$. Для каждого $n \in \mathbb{N}$ определим непрерывную функцию на $\Omega_{1}^{n} \times \Omega_{2}^{n} \times \Omega_{2}$ формулой

$$
K_{n}^{(1)}\left(\begin{array}{c}
x_{1}, \ldots, x_{n} ; y \\
s_{1}, \ldots, s_{n} ; \lambda
\end{array}\right)=\left|\begin{array}{ccc}
h_{1}\left(x_{1}, s_{1}, y ; \lambda\right) & \ldots & h_{1}\left(x_{1}, s_{n}, y ; \lambda\right) \\
\vdots & \ddots & \vdots \\
h_{1}\left(x_{n}, s_{1}, y ; \lambda\right) & \ldots & h_{1}\left(x_{n}, s_{n}, y ; \lambda\right)
\end{array}\right|
$$

где

$$
h_{1}(x, s, y ; \lambda)=\frac{k_{1}(x, s, y)}{k_{0}(s, y)-\lambda}, \quad \lambda \in \rho\left(H_{0}\right)
$$


На $\Omega_{1}^{n} \times \Omega_{2}^{n} \times \Omega_{1}$ аналогично определим функцию

$$
K_{n}^{(2)}\left(\begin{array}{c}
y_{1}, \ldots, y_{n} ; x \\
t_{1}, \ldots, t_{n} ; \lambda
\end{array}\right)=\left|\begin{array}{ccc}
h_{2}\left(x, t_{1}, y_{1} ; \lambda\right) & \ldots & h_{2}\left(x, t_{n}, y_{1} ; \lambda\right) \\
\vdots & \ddots & \vdots \\
h_{2}\left(x, t_{1}, y_{n} ; \lambda\right) & \ldots & h_{2}\left(x, t_{n}, y_{n} ; \lambda\right)
\end{array}\right|
$$

где

$$
h_{2}(x, t, y ; \lambda)=\frac{k_{2}(x, t, y)}{k_{0}(x, t)-\lambda}, \quad \lambda \in \rho\left(H_{0}\right) .
$$

Для каждого значения $\lambda \in \rho\left(H_{0}\right)$ определим последовательности непрерывных функций $d_{n}^{(1)}(y ; \lambda)$ на $\Omega_{2}$ и $d_{n}^{(2)}(x ; \lambda)$ на $\Omega_{1}(n \in \mathbb{N})$ следующим образом:

$$
d_{n}^{(k)}(\xi ; \lambda)=\int_{\Omega_{k}} \ldots \int_{\Omega_{k}} K^{(k)}\left(\begin{array}{l}
\xi_{1}, \ldots, \xi_{n} ; \xi \\
\xi_{1}, \ldots, \xi_{n} ; \lambda
\end{array}\right) d \xi_{1} \ldots d \xi_{n}, \quad k=1,2 .
$$

Детерминанты $D_{1}(y ; z, \varepsilon)$ и $D_{2}(x ; z, \varepsilon)$ имеют следующие формы [13], [14]:

$$
D_{1}(y ; \lambda, \varepsilon)=1+\sum_{n \in N} \frac{(-\varepsilon)^{n}}{n !} d_{n}^{(1)}(y ; \lambda), \quad D_{2}(x ; \lambda, \varepsilon)=1+\sum_{n \in N} \frac{(-\varepsilon)^{n}}{n !} d_{n}^{(2)}(x ; \lambda) .
$$

Обозначим через $M_{1}(x, s, y ; z, \varepsilon)$ и $M_{2}(x, t, y ; z, \varepsilon)$ соответственно миноры операторов $E-\varepsilon W_{1}(z)$ и $E-\varepsilon W_{2}(z), z \in \rho\left(H_{0}\right)$. Тогда [13], [14]

$$
\begin{aligned}
& M_{1}(x, s, y ; \lambda, \varepsilon)=h_{1}(x, s, y ; \lambda)+\sum_{n=1}^{\infty} \frac{(-\varepsilon)^{n}}{n !} q_{n}^{(1)}(x, s, y ; \lambda), \\
& M_{2}(x, t, y ; \lambda, \varepsilon)=h_{2}(x, t, y ; \lambda)+\sum_{n=1}^{\infty} \frac{(-\varepsilon)^{n}}{n !} q_{n}^{(2)}(x, t, y ; \lambda),
\end{aligned}
$$

где

$$
\begin{aligned}
& q_{n}^{(1)}(x, s, y ; \lambda)=\int_{\Omega_{1}} \ldots \int_{\Omega_{1}} K_{n+1}^{(1)}\left(\begin{array}{c}
x, s_{1} \ldots, s_{n} ; y \\
s, s_{1}, \ldots, s_{n} ; \lambda
\end{array}\right) d s_{1} \ldots d s_{n} \\
& q_{n}^{(2)}(x, t, y ; \lambda)=\int_{\Omega_{2}} \ldots \int_{\Omega_{2}} K_{n+1}^{(2)}\left(\begin{array}{c}
y, t_{1} \ldots, t_{n} ; x \\
t, t_{1}, \ldots, t_{n} ; \lambda
\end{array}\right) d t_{1} \ldots d t_{n} .
\end{aligned}
$$

Пусть $\lambda \in \mathbb{C} \backslash \sigma\left(H_{1}(\varepsilon)\right)$. Тогда оператор $E-\varepsilon W_{1}(\lambda)$ обратим и обратный оператор $\left(E-\varepsilon W_{1}(\lambda)\right)^{-1}$ является ограниченным, при этом $\left(E-\varepsilon W_{1}(\lambda)\right)^{-1}=E+\varepsilon P_{1}(\lambda, \varepsilon)$, где $P_{1}(\lambda, \varepsilon)$ - ЧИО, заданный равенством

$$
P_{1}(\lambda, \varepsilon) f(x, y)=\int_{\Omega_{1}} \frac{M_{1}(x, s, y ; \lambda, \varepsilon)}{D_{1}(y ; \lambda, \varepsilon)} f(s, y) d s .
$$

Аналогично, если $\lambda \in \mathbb{C} \backslash \sigma\left(H_{2}(\varepsilon)\right)$, то $E-\varepsilon W_{2}(\lambda)$ обратим и обратный оператор $\left(E-\varepsilon W_{2}(\lambda)\right)^{-1}$ является ограниченным, при этом $\left(E-\varepsilon W_{2}(\lambda)\right)^{-1}=E+\varepsilon P_{2}(\lambda, \varepsilon)$, где $P_{2}(\lambda, \varepsilon)$ - ЧИО, заданный равенством

$$
P_{2}(\lambda, \varepsilon) f(x, y)=\int_{\Omega_{2}} \frac{M_{2}(x, t, y ; \lambda, \varepsilon)}{D_{2}(x ; \lambda, \varepsilon)} f(x, t) d t
$$


Для каждого $\lambda \in \mathbb{C} \backslash\left\{\sigma\left(H_{1}(\varepsilon)\right) \cup \sigma\left(H_{2}(\varepsilon)\right)\right\}$ в пространстве $L_{2}\left(\Omega_{1} \times \Omega_{2}\right)$ определим компактный оператор $K(\lambda, \varepsilon)$ следующим образом:

$$
K(\lambda, \varepsilon)=\left(E-\varepsilon W_{2}(\lambda)\right)^{-1} P_{1}(\lambda, \varepsilon) W_{2}(\lambda)
$$

Обозначим через $\Delta(\lambda, \varepsilon)$ определитель Фредгольма оператора $E-\varepsilon K(\lambda, \varepsilon)$. Для дискретного спектра трехчастичного оператора $H(\varepsilon)$ имеет место следующее равенство [7]:

$$
\sigma_{\text {disc }}(H(\varepsilon))=\left\{\lambda \in \mathbb{C} \backslash\left\{\sigma\left(H_{1}(\varepsilon)\right) \cup \sigma\left(H_{2}(\varepsilon)\right)\right\}: \Delta(\lambda, \varepsilon)=0\right\}
$$

Положим

$$
m=\inf _{(x, y) \in \Omega_{1} \times \Omega_{2}} k_{0}(x, y), \quad M=\sup _{(x, y) \in \Omega_{1} \times \Omega_{2}} k_{0}(x, y) .
$$

Допустим, что

$m=k_{0}\left(x_{\min }, y_{\min }\right), \quad M=k_{0}\left(x_{\max }, y_{\max }\right), \quad\left(x_{\min }, y_{\min }\right),\left(x_{\max }, y_{\max }\right) \in \Omega_{1} \times \Omega_{2}$.

На множестве $\Omega_{2}$ определим следующие функции:

$$
F_{1}(y)=\lim _{\lambda \rightarrow m-0} \int_{\Omega_{1}} \frac{d s}{k_{0}(s, y)-\lambda}, \quad F_{2}(y)=\lim _{\lambda \rightarrow M+0} \int_{\Omega_{1}} \frac{d s}{\lambda-k_{0}(s, y)} .
$$

Аналогично на множестве $\Omega_{1}$ определим следующие функции

$$
G_{1}(x)=\lim _{\lambda \rightarrow m-0} \int_{\Omega_{2}} \frac{d t}{k_{0}(x, t)-\lambda}, \quad G_{2}(x)=\lim _{\lambda \rightarrow M+0} \int_{\Omega_{2}} \frac{d t}{\lambda-k_{0}(x, t)} .
$$

Пусть значения $F_{1}\left(y_{\min }\right), F_{2}\left(y_{\max }\right), G_{1}\left(x_{\min }\right)$ и $G_{2}\left(x_{\max }\right)$ являются конечными. Тогда функции $F_{1}(y), F_{2}(y), G_{1}(x)$ и $G_{2}(x)$ непрерывны и имеют место следующие неравенства:

$$
\begin{aligned}
& 0 \leqslant \int_{\Omega_{1}} \frac{d s}{k_{0}(s, y)-\lambda} \leqslant F_{1}(y) \quad \text { при } \lambda \leqslant m, \\
& 0 \leqslant \int_{\Omega_{1}} \frac{d s}{\lambda-k_{0}(s, y)} \leqslant F_{2}(y) \quad \text { при } \lambda \geqslant M,
\end{aligned}
$$

где $y \in \Omega_{2}$;

$$
\begin{aligned}
& 0 \leqslant \int_{\Omega_{2}} \frac{d t}{k_{0}(x, t)-\lambda} \leqslant G_{1}(x) \quad \text { при } \lambda \leqslant m, \\
& 0 \leqslant \int_{\Omega_{2}} \frac{d t}{\lambda-k_{0}(x, t)} \leqslant G_{2}(x) \quad \text { при } \lambda \geqslant M,
\end{aligned}
$$

где $x \in \Omega_{1}$.

Положим

$$
J_{k}=\max _{y \in \Omega_{2}} F_{k}(y), \quad I_{k}=\max _{x \in \Omega_{1}} G_{k}(x), \quad k=1,2 .
$$

ТЕОРема 2.1. Пусть выполнено неравенство

$$
\varepsilon<\frac{1}{2 k_{1}^{\max }} \frac{1}{\max \left\{J_{1}, J_{2}\right\}} \quad\left(\varepsilon<\frac{1}{2 k_{2}^{\max }} \frac{1}{\max \left\{I_{1}, I_{2}\right\}}\right),
$$


¿əe

$$
k_{1}^{\max }=\sup _{(x, s, y) \in \Omega_{1}^{2} \times \Omega_{2}}\left|k_{1}(x, s, y)\right|, \quad k_{2}^{\max }=\sup _{(x, t, y) \in \Omega_{1} \times \Omega_{2}^{2}}\left|k_{2}(x, t, y)\right| .
$$

Тогда для спектра оператора $H_{1}(\varepsilon)=H_{0}-\varepsilon T_{1}$ (оператора $H_{2}(\varepsilon)=H_{0}-\varepsilon T_{2}$ ) имеет место равенство $\sigma\left(H_{1}(\varepsilon)\right)=\sigma\left(H_{0}\right)$ (равенство $\left.\sigma\left(H_{2}(\varepsilon)\right)=\sigma\left(H_{0}\right)\right)$, т.е. в спектре оператора $H_{1}(\varepsilon)$ (оператора $H_{1}(\varepsilon)$ ) отсутствует дополнительная часть $\sigma_{1}(\varepsilon)$ (дополнительная часть $\left.\sigma_{2}(\varepsilon)\right)$.

ТеОрема 2.2. Если выполнено неравенство

$$
\varepsilon<\mu_{0}=\frac{1}{2} \min \left\{\frac{1}{k_{1}^{\max } J_{0}}, \frac{1}{k_{2}^{\max } I_{0}}\right\}
$$

где $J_{0}=\max \left\{J_{1}, J_{2}\right\}, I_{0}=\max \left\{I_{1}, I_{2}\right\}$, то для существенного спектра трехчастиного оператора $H(\varepsilon)$ имеет место равенство

$$
\sigma_{\mathrm{ess}}(H(\varepsilon))=\sigma_{\mathrm{ess}}(H(0))=\sigma\left(H_{0}\right),
$$

m.е. в спектре оператора $H(\varepsilon)$ отсутствует двухчастичная ветвъ.

На множестве $\Omega_{1}$ определим следующие функции:

$$
\psi_{1}(x)=\lim _{\lambda \rightarrow m-0} \int_{\Omega_{2}} \frac{d t}{\left(k_{0}(x, t)-\lambda\right)^{4}}, \quad \psi_{2}(x)=\lim _{\lambda \rightarrow M+0} \int_{\Omega_{2}} \frac{d t}{\left(k_{0}(x, t)-\lambda\right)^{4}} .
$$

Допустим, что $\psi_{1}\left(x_{\min }\right)$ и $\psi_{2}\left(x_{\max }\right)$ конечны. Тогда функции $\psi_{1}(x)$ и $\psi_{2}(x)$ являются непрерывными на $\Omega_{1}$ и справедливы следующие неравенства:

$$
\begin{aligned}
& 0 \leqslant \int_{\Omega_{2}} \frac{d t}{\left(k_{0}(x, t)-\lambda\right)^{4}} \leqslant \psi_{1}(x) \quad \text { при } \lambda \leqslant m, \\
& 0 \leqslant \int_{\Omega_{2}} \frac{d t}{\left(k_{0}(x, t)-\lambda\right)^{4}} \leqslant \psi_{2}(x) \quad \text { при } \lambda \geqslant M,
\end{aligned}
$$

где $x \in \Omega_{1}$.

Положим

$$
\Lambda_{k}=\sup _{x \in \Omega_{1}} \psi_{k}(x), \quad k=1,2, \quad \Lambda_{0}=\max \left\{\Lambda_{1}, \Lambda_{2}\right\} .
$$

Пусть $a \in\left(0, \mu_{0}\right)$. Определим величину

где

$$
\gamma(a)=\frac{\varphi_{1}(a) \varphi_{2}(a)}{3 k_{1}^{\max } k_{2}^{\max }\left(2+3 \sqrt[4]{\Lambda_{0}} k_{2}^{\max }\right) \sqrt{\Lambda_{0}}}
$$

$$
\varphi_{1}(a)=1-\frac{a J_{0} k_{1}^{\max }}{1-a J_{0} k_{1}^{\max }}, \quad \varphi_{2}(a)=1-\frac{a I_{0} k_{2}^{\max }}{1-a I_{0} k_{2}^{\max }} .
$$

Для каждого $a \in\left(0, \mu_{0}\right)$ определим также положительное число $\gamma_{0}(a)$ :

$$
\gamma_{0}(a)= \begin{cases}a, & \text { если } \mu_{0} \leqslant \gamma(a) \\ \gamma(a), & \text { если } \mu_{0}>\gamma(a) .\end{cases}
$$

TeOpema 2.3. Пусть $a \in\left(0, \mu_{0}\right)$. При всех

$$
\varepsilon \in\left(0, \gamma_{0}(a)\right]
$$

в спектре трехчастичного оператора $H(\varepsilon)$ отсутствуют двухчастичная ветвъ и связанные состояния, т.е. $\sigma_{\mathrm{ess}}(H(\varepsilon))=\sigma(H(0)) u \sigma_{\mathrm{disc}}(H(\varepsilon))=\varnothing$. 


\section{3. ВСПОМОГАТЕЛЬНЫЕ ЛЕММЫ}

ЛЕмма 3.1. Если выполнено неравенство

$$
\varepsilon<\frac{1}{2 k_{1}^{\max }} \frac{1}{J_{0}} \quad \text { или } \quad \varepsilon<\frac{1}{2 k_{2}^{\max }} \frac{1}{I_{0}}
$$

то $\sigma_{1}(\varepsilon)=\varnothing$ или $\sigma_{2}(\varepsilon)=\varnothing$ соответственно.

ДокАЗАтЕльство. Докажем лемму для множества $\sigma_{1}(\varepsilon)$, доказательство для множества $\sigma_{2}(\varepsilon)$ можно провести аналогично. Как известно, спектр самосопряженных операторов лежит на вещественной оси. Отсюда и из определения (2.1) следует, что нам достаточно проанализировать функцию $D_{1}(y ; \lambda, \varepsilon)$ при $\lambda \in \mathbb{R} \backslash[m, M]$. В этом случае для детерминанта $D_{1}(y ; \lambda, \varepsilon)$ имеем

$$
D_{1}(y ; \lambda, \varepsilon)=1-\varepsilon \Delta_{1}(y ; \lambda, \varepsilon),
$$

где

$$
\Delta_{1}(y ; \lambda, \varepsilon)=\sum_{n=1}^{\infty} \frac{(-\varepsilon)^{n-1}}{n !} d_{n}^{(1)}(y ; \lambda)
$$

Для функции $K_{n}^{(1)}$ справедливо равенство

$$
K_{n}^{(1)}\left(\begin{array}{c}
x_{1}, \ldots, x_{n} ; y \\
s_{1}, \ldots, s_{n} ; \lambda
\end{array}\right)=\frac{1}{\prod_{j=1}^{n}\left(k_{0}\left(s_{j} ; y\right)-\lambda\right)} \Pi_{n}^{(1)}\left(\begin{array}{c}
x_{1}, \ldots, x_{n} ; y \\
s_{1}, \ldots, s_{n} ; y
\end{array}\right),
$$

где

$$
\Pi_{n}^{(1)}\left(\begin{array}{c}
x_{1}, \ldots, x_{n} ; y \\
s_{1}, \ldots, s_{n} ; y
\end{array}\right)=\left|\begin{array}{ccc}
k_{1}\left(x_{1}, s_{1}, y\right) & \ldots & k_{1}\left(x_{1}, s_{n}, y\right) \\
\vdots & \ddots & \vdots \\
k_{1}\left(x_{n}, s_{1}, y\right) & \ldots & k_{1}\left(x_{n}, s_{n}, y\right)
\end{array}\right| .
$$

Пользуясь неравенством Адамара, получим

$$
\left|\Pi_{n}^{(1)}\left(\begin{array}{c}
x_{1}, \ldots, x_{n} ; y \\
s_{1}, \ldots, s_{n} ; y
\end{array}\right)\right| \leqslant\left(k_{1}^{\max }\right)^{n} n^{n / 2}, \quad n \in \mathbb{N} .
$$

При $\lambda \in \mathbb{R} \backslash[m, M]$ имеем

$$
\left|d_{n}^{(1)}(y ; \lambda)\right| \leqslant\left(k_{1}^{\max } n^{1 / 2} \int_{\Omega_{1}} \frac{d s}{\left|k_{0}(s, y)-\lambda\right|}\right)^{n} \leqslant\left(J_{0} k_{1}^{\max } n^{1 / 2}\right)^{n}, \quad n \in \mathbb{N} .
$$

Отсюда, учитывая равенство

$$
\sup _{n \in \mathbb{N}} \frac{n^{n / 2}}{n !}=1
$$

получим, что

$$
\left|\Delta_{1}(y ; \lambda, \varepsilon)\right| \leqslant \sum_{n=1}^{\infty} \frac{\varepsilon^{n-1}}{n !}\left(J_{0} k_{1}^{\max }\right)^{n} n^{n / 2}<\sum_{n=1}^{\infty}\left(J_{0} k_{1}^{\max }\right)^{n} \varepsilon^{n-1}=\frac{J_{0} k_{1}^{\max }}{1-\varepsilon J_{0} k_{1}^{\max }},
$$

так как $\varepsilon J_{0} k_{1}^{\max }<1$ в силу неравенства (3.1). 
Таким образом,

$$
\varepsilon\left|\Delta_{1}(y ; \lambda, \varepsilon)\right| \leqslant \frac{\varepsilon J_{0} k_{1}^{\max }}{1-\varepsilon J_{0} k_{1}^{\max }}<1 \quad \text { при всех } y \in \Omega_{2} \text { и } \lambda \in \mathbb{R} \backslash[m, M] .
$$

Из последнего неравенства и из формулы (3.2) вытекает, что $D_{1}(y ; \lambda, \varepsilon)>0$ при всех $y \in \Omega_{2}$ и любых $\lambda \in \mathbb{R} \backslash[m, M]$. Отсюда и из $(2.1)$ следует, то $\sigma_{1}(\varepsilon)=\varnothing$. Лемма доказана.

Пусть $a_{0} \in\left(0,1 /\left(2 k_{1}^{\max } J_{0}\right)\right)$ - фиксированное число и

$$
\Gamma_{1}=\Omega_{2} \times(-\infty, m) \times\left(0, a_{0}\right], \quad \Gamma_{2}=\Omega_{2} \times(M,+\infty) \times\left(0, a_{0}\right] .
$$

Лемма 3.2. Справедливы неравенства

$$
\inf _{(y, \lambda, \varepsilon) \in \Gamma_{1} \cup \Gamma_{2}} D_{1}(y ; \lambda, \varepsilon)=D_{1}^{\min }>0, \quad \sup _{(y, \lambda, \varepsilon) \in \Gamma_{1} \cup \Gamma_{2}} D_{1}(y ; \lambda, \varepsilon)=D_{1}^{\max } \leqslant 2 .
$$

ДоказАтельство. Имеем $D_{1}(y ; \lambda, \varepsilon)=1-\varepsilon \Delta_{1}(y ; \lambda, \varepsilon)$ при всех $(y, \lambda, \varepsilon) \in \Gamma_{1} \cup \Gamma_{2}$ и в силу неравенства (3.2) получим, что $0<D_{1}(y ; \lambda, \varepsilon)<2$ при всех $(y, \lambda, \varepsilon) \in \Gamma_{1} \cup \Gamma_{2}$. Отсюда

$$
D_{1}^{\max }=\sup _{(y, \lambda, \varepsilon) \in \Gamma_{1} \cup \Gamma_{2}} D_{1}(y ; \lambda, \varepsilon) \leqslant 2
$$

и

$$
\begin{aligned}
\inf _{(y, \lambda, \varepsilon) \in \Gamma_{1} \cup \Gamma_{2}} D_{1}(y ; \lambda, \varepsilon) & \geqslant 1-\sup _{(y, \lambda, \varepsilon) \in \Gamma_{1} \cup \Gamma_{2}} \varepsilon\left|\Delta_{1}(y ; \lambda, \varepsilon)\right| \geqslant \\
& \geqslant 1-\sup _{\varepsilon \in(0, a]} \frac{\varepsilon J_{0} k_{1}^{\max }}{1-\varepsilon J_{0} k_{1}^{\max }}=1-\frac{a J_{0} k_{1}^{\max }}{1-a J_{0} k_{1}^{\max }}>0 .
\end{aligned}
$$

Лемма доказана.

Положим

$$
\Gamma_{1}^{\prime}=\Omega_{1} \times(-\infty, m) \times\left(0, b_{0}\right], \quad \Gamma_{2}^{\prime}=\Omega_{1} \times(M,+\infty) \times\left(0, b_{0}\right],
$$

где $b_{0} \in\left(0,1 /\left(2 k_{2}^{\max } I_{0}\right)\right)$ - фиксированное число. Аналогично лемме 3.2 доказывается следующее утверждение.

ЛЕмма 3.3. Справедливы неравенства

$$
\inf _{(x, \lambda, \varepsilon) \in \Gamma_{1}^{\prime} \cup \Gamma_{2}^{\prime}} D_{2}(x ; \lambda, \varepsilon)=D_{2}^{\min }>0, \quad \sup _{(x, \lambda, \varepsilon) \in \Gamma_{1}^{\prime} \cup \Gamma_{2}^{\prime}} D_{2}(x ; \lambda, \varepsilon)=D_{2}^{\max } \leqslant 2 .
$$

ЛЕмма 3.4. Если $\varepsilon<1 / J_{0} k_{1}^{\max }$, то для каждого $\lambda \in \rho\left(H_{0}\right)$

$$
\left|M_{1}(x, s, y ; \lambda, \varepsilon)\right| \leqslant \Phi_{1}(\varepsilon) \frac{k_{1}^{\max }}{\left|k_{0}(s, y)-\lambda\right|}, \quad(x, s, y) \in \Omega_{1}^{2} \times \Omega_{2},
$$

где

$$
\Phi_{1}(\varepsilon)=1+\sum_{k=1}^{\infty}\left(\varepsilon J_{0} k_{1}^{\max }\right)^{k} \frac{(k+1)^{(k+1) / 2}}{k !} .
$$


ДокАЗАТЕЛЬСтво. Для $\lambda \in \rho\left(H_{0}\right)$ имеем

$$
\begin{aligned}
& K_{n+1}^{(1)}\left(\begin{array}{l}
x, s_{1} \ldots, s_{n} ; y \\
s, s_{1}, \ldots, s_{n} ; \lambda
\end{array}\right)= \\
& \quad=\frac{1}{\left(k_{0}(s, y)-\lambda\right) \prod_{j=1}^{n}\left(k_{0}\left(s_{j}, y\right)-\lambda\right)}\left|\begin{array}{ccc}
k_{1}(x, s, y) & \ldots & k_{1}\left(x, s_{n}, y\right) \\
k_{1}\left(s_{1}, s, y\right) & \ldots & k_{1}\left(s_{1}, s_{n} ; y\right) \\
\vdots & \ddots & \vdots \\
k_{1}\left(s_{n}, s, y\right) & \ldots & k_{1}\left(s_{n}, s_{n}, y\right)
\end{array}\right| .
\end{aligned}
$$

Отсюда и в силу неравенства Адамара получим

$$
\left|K_{n+1}^{(1)}\left(\begin{array}{l}
x, s_{1} \ldots, s_{n} ; y \\
s, s_{1}, \ldots, s_{n} ; \lambda
\end{array}\right)\right|=\frac{\left(k_{1}^{\max }\right)^{n+1}(n+1)^{(n+1) / 2}}{\left|k_{0}(s, y)-\lambda\right| \prod_{j=1}^{n}\left|k_{0}\left(s_{j}, y\right)-\lambda\right|} .
$$

Тогда

$$
\left|q_{n}^{(1)}(x, s, y ; \lambda)\right| \leqslant \frac{J_{0}^{n}\left(k_{1}^{\max }\right)^{n+1}(n+1)^{(n+1) / 2}}{\left|k_{0}(s, y)-\lambda\right|} .
$$

Следовательно,

$$
\begin{aligned}
\left|M_{1}(x, s, y ; \lambda, \varepsilon)\right| & \leqslant \frac{k_{1}^{\max }}{\left|k_{0}(s, y)-\lambda\right|}+\sum_{n=1}^{\infty} \frac{\varepsilon^{n} J_{0}^{n}\left(k_{1}^{\max }\right)^{n+1}(n+1)^{(n+1) / 2}}{n !\left|k_{0}(s, y)-\lambda\right|}= \\
& =\frac{k_{1}^{\max }}{\left|k_{0}(s, y)-\lambda\right|}\left(1+\sum_{n=1}^{\infty}\left(\varepsilon J_{0} k_{1}^{\max }\right)^{n} \frac{(n+1)^{(n+1) / 2}}{n !}\right)= \\
& =\Phi_{1}(\varepsilon) \frac{k_{1}^{\max }}{\left|k_{0}(s, y)-\lambda\right|} .
\end{aligned}
$$

Аналогично лемме 3.4 доказывается следующее утверждение.

Лемма 3.5. Если $\varepsilon<1 / I_{0} k_{2}^{\max }$, то для каждого $\lambda \in \rho\left(H_{0}\right)$

$$
\left|M_{2}(x, t, y ; \lambda, \varepsilon)\right| \leqslant \Phi_{2}(\varepsilon) \frac{k_{2}^{\max }}{\left|k_{0}(t, x)-\lambda\right|}, \quad(x, t, y) \in \Omega_{1} \times \Omega_{2}^{2},
$$

где

$$
\Phi_{2}(\varepsilon)=1+\sum_{n=1}^{\infty}\left(\varepsilon I_{0} k_{2}^{\max }\right)^{n} \frac{(n+1)^{(n+1) / 2}}{n !} .
$$

Лемма 3.6. Пусть $\lambda \in \rho\left(H_{0}\right)$ u $0<a<\mu_{0}$. Тогда при $0<\varepsilon \leqslant a$ для ядра $q(x, y ; s, t ; \lambda, \varepsilon)$ интегрального оператора $K(\lambda, \varepsilon)$ имеет место неравенство

$$
|q(x, y ; s, t ; \lambda, \varepsilon)| \leqslant A_{1}(\varepsilon)\left(\frac{1}{\left(k_{0}(s, y)-\lambda\right)^{2}}+A_{2}(\varepsilon)\right),
$$

где

$$
A_{1}(\varepsilon)=\frac{k_{1}^{\max } k_{2}^{\max }}{D_{1}^{\min }} \Phi_{1}(\varepsilon), \quad A_{2}(\varepsilon)=\frac{\Lambda_{0}^{3 / 4} k_{2}^{\max }}{D_{2}^{\min }} \Phi_{2}(\varepsilon) .
$$


ДокАЗАТЕЛЬСТво. В силу лемм 3.1 и 3.2 имеем $\sigma_{1}(\varepsilon)=\sigma_{2}(\varepsilon)=\varnothing$ (см. равенства (2.1) и (2.2)). Следовательно, $\sigma_{\text {ess }}(H(\varepsilon))=\sigma\left(H_{0}\right)$. Пусть $\lambda \in \rho\left(H_{0}\right)$. Ядро $q(x, y ; s, t ; \lambda, \varepsilon)$ компактного оператора $K(\lambda, \varepsilon)$ записывается как [7]

$$
\begin{aligned}
q(x, y ; s, t ; \lambda, \varepsilon)= & \frac{M_{1}(x, s, y ; \lambda, \varepsilon)}{D_{1}(y ; \lambda, \varepsilon)} \frac{k_{2}(s, t, y)}{k_{0}(s, y)-\lambda}+ \\
& +\int_{\Omega_{2}} \frac{M_{2}(x, \xi, y ; \lambda, \varepsilon)}{D_{2}(x ; \lambda, \varepsilon)} \frac{M_{1}(x, s, \xi ; \lambda, \varepsilon)}{D_{1}(\xi ; \lambda, \varepsilon)} \frac{k_{2}(s, t, \xi)}{k_{0}(s, \xi)-\lambda} d \xi .
\end{aligned}
$$

Отсюда вытекает, что

$$
\begin{aligned}
& |q(x, y ; s, t ; \lambda, \varepsilon)| \leqslant \frac{k_{1}^{\max } k_{2}^{\max }}{D_{1}^{\min }} \frac{\Phi_{1}(\varepsilon)}{\left(k_{0}(s, y)-\lambda\right)^{2}}+ \\
& \quad+\frac{k_{1}^{\max }\left(k_{2}^{\max }\right)^{2}}{D_{1}^{\min } D_{2}^{\min }} \Phi_{1}(\varepsilon) \Phi_{2}(\varepsilon) \int_{\Omega_{2}} \frac{1}{\left|k_{0}(x, \xi)-\lambda\right|} \frac{d \xi}{\left(k_{0}(s, \xi)-\lambda\right)^{2}}= \\
& \quad=\frac{k_{1}^{\max } k_{2}^{\max }}{D_{1}^{\min }} \Phi_{1}(\varepsilon)\left(\frac{1}{\left(k_{0}(s, y)-\lambda\right)^{2}}+\frac{k_{2}^{\max }}{D_{2}^{\min }} \Phi_{2}(\varepsilon) \int_{\Omega_{2}} \frac{d \xi}{\left|k_{0}(x, \xi)-\lambda\right|\left(k_{0}(s, \xi)-\lambda\right)^{2}}\right) \leqslant \\
& \leqslant
\end{aligned}
$$

где $\Lambda_{0}=\max \left\{\Lambda_{1}, \Lambda_{2}\right\}$.

\section{4. ДОКАЗАТЕЛЬСТВО ОСНОВНОЙ ТЕОРЕМЫ}

Из равенств (2.1)-(2.3) с учетом лемм 3.1, 3.2 вытекает теорема 2.1. Из теоремы 2.1 с учетом равенства (2.4) вытекает теорема 2.2 .

Пусть для $\varepsilon>0$ выполнены неравенства теоремы 2.1. Тогда

$$
\sigma_{\text {ess }}(H(\varepsilon))=\sigma(H(0))=\sigma\left(H_{0}\right) .
$$

Для каждого $\varepsilon$ и $z \in \rho\left(H_{0}\right)$ определим функцию $\Pi_{n}$ на $\left(\Omega_{1} \times \Omega_{2}\right)^{n} \times\left(\Omega_{1} \times \Omega_{2}\right)^{n}$ :

$$
\Pi_{n}\left(\begin{array}{c}
\omega_{1}, \ldots, \omega_{n} ; \varepsilon \\
\omega_{1}^{\prime}, \ldots, \omega_{n}^{\prime} ; z
\end{array}\right)=\left|\begin{array}{ccc}
q\left(\omega_{1}, \omega_{1}^{\prime} ; z, \varepsilon\right) & \ldots & q\left(\omega_{1}, \omega_{n}^{\prime} ; z, \varepsilon\right) \\
\vdots & \ddots & \vdots \\
q\left(\omega_{n}, \omega_{1}^{\prime} ; z, \varepsilon\right) & \ldots & q\left(\omega_{n}, \omega_{n}^{\prime} ; z, \varepsilon\right)
\end{array}\right|,
$$

где $\omega_{k}=\left(x_{k}, y_{k}\right) \in \Omega_{1} \times \Omega_{2}, \omega_{k}^{\prime}=\left(s_{k}, t_{k}\right) \in \Omega_{1} \times \Omega_{2}$. Тогда для детерминанта Фредгольма $\Delta(\lambda, \varepsilon)$ оператора $E-\varepsilon K(\lambda, \varepsilon), \lambda \in \rho\left(H_{0}\right)$, имеет место формула

$$
\Delta(\lambda, \varepsilon)=1+\sum_{n=1}^{\infty} \frac{(-\varepsilon)^{n}}{n !} u_{n}(\lambda, \varepsilon),
$$

где

$$
u_{n}(\lambda, \varepsilon)=\int_{\Omega_{1} \times \Omega_{2}} \ldots \int_{\Omega_{1} \times \Omega_{2}} \Pi_{n}\left(\begin{array}{c}
\omega_{1}^{\prime}, \ldots, \omega_{n}^{\prime} ; \varepsilon \\
\omega_{1}^{\prime}, \ldots, \omega_{n}^{\prime} ; \lambda
\end{array}\right) d \omega_{1}^{\prime} \ldots d \omega_{n}^{\prime} .
$$

Tеорема 4.1. Если при $\varepsilon \in\left(0, \mu_{0}\right)$ выполнено неравенство

$$
\varepsilon<\frac{1}{2 A_{1}(\varepsilon)\left(\sqrt{\Lambda_{0}}+A_{2}(\varepsilon)\right)},
$$

mо у оператора $H(\varepsilon)$ отсутствует дискретный спектр. 
ДоказАтельство. Пусть $\varepsilon \in\left(0, \mu_{0}\right)$. Тогда $\sigma_{\mathrm{ess}}(H(\varepsilon))=\sigma\left(H_{0}\right)$ в силу теоремы 2.2. Положим

$$
q_{i, j}(\lambda, \varepsilon)=q\left(s_{i}, t_{i} ; s_{j}, t_{j} ; \lambda, \varepsilon\right), \quad i, j \in \mathbb{N} .
$$

Из определения детерминанта $n$-го порядка имеем

$$
\Pi_{n}\left(\begin{array}{c}
\omega_{1}, \ldots, \omega_{n} ; \varepsilon \\
\omega_{1}^{\prime}, \ldots, \omega_{n}^{\prime} ; \lambda
\end{array}\right)=\sum_{\left(j_{1}, \ldots, j_{n}\right)}(-1)^{k} q_{1, j_{1}}(\lambda, \varepsilon), q_{2, j_{2}}(\lambda, \varepsilon), \ldots, q_{n, j_{n}}(\lambda, \varepsilon),
$$

где сумма состоит из $n$ ! членов вида $(-1)^{k} q_{1, j_{1}}, \ldots, q_{n, j_{n}}$, каждый из которых соответствует одному из $n$ ! различных упорядоченных множеств $\left\{j_{1}, \ldots, j_{n}\right\}$, полученных $k$ попарными перестановками элементов из множества $\{1,2, \ldots, n\}$. Следовательно, пользуясь леммой 3.5 , получим

$$
\begin{aligned}
\left|\Pi_{n}\left(\begin{array}{c}
\omega_{1}, \ldots, \omega_{n} ; \varepsilon \\
\omega_{1}^{\prime}, \ldots, \omega_{n}^{\prime} ; \lambda
\end{array}\right)\right| & \leqslant \sum_{\left(j_{1}, \ldots, j_{n}\right)}\left|q_{1, j_{1}}(\lambda, \varepsilon)\right| \ldots\left|q_{n, j_{n}}(\lambda, \varepsilon)\right| \leqslant \\
& \leqslant \sum_{\left(j_{1}, \ldots, j_{n}\right)} \varepsilon^{n} A_{1}^{n}(\varepsilon) \prod_{j=1}^{n}\left(\frac{1}{\left(k_{0}\left(s_{j_{i}}, t_{i}\right)-\lambda\right)^{2}}+A_{2}(\varepsilon)\right) .
\end{aligned}
$$

Отсюда

$$
\begin{aligned}
\left|u_{n}(\lambda, \varepsilon)\right| & \leqslant \int_{\Omega_{1} \times \Omega_{2}} \ldots \int_{\Omega_{1} \times \Omega_{2}} \Pi_{n}\left(\begin{array}{l}
\omega_{1}^{\prime}, \ldots, \omega_{n}^{\prime} ; \varepsilon \\
\omega_{1}^{\prime}, \ldots, \omega_{n}^{\prime} ; \lambda
\end{array}\right) d \omega_{1}^{\prime} \ldots d \omega_{n}^{\prime} \leqslant \\
& \leqslant \sum_{\left(j_{1}, \ldots, j_{n}\right)}\left(\varepsilon A_{1}(\varepsilon)\right)^{n} \int_{\Omega_{1}} \ldots \int_{\Omega_{1}} \prod_{i=1}^{n}\left(\int_{\Omega_{2}} \frac{d t_{i}}{\left(k_{0}\left(s_{j_{i}}, t_{i}\right)-\lambda\right)^{2}}+A_{2}(\varepsilon)\right) d s_{1} \ldots d s_{n} \leqslant \\
& \leqslant\left(\varepsilon^{n} A_{1}(\varepsilon)\right)^{n} \sum_{\left(j_{1}, \ldots, j_{n}\right)} \int_{\Omega_{1}} \ldots \int_{\Omega_{1}}\left(\sqrt{\Lambda_{0}}+A_{2}(\varepsilon)\right)^{n} d s_{1} \ldots d s_{n}= \\
& =n !\left[\varepsilon A_{1}(\varepsilon)\left(\sqrt{\Lambda_{0}}+A_{2}(\varepsilon)\right]^{n}, \quad n \in \mathbb{N} .\right.
\end{aligned}
$$

Рассмотрим функцию

$$
\Delta_{0}(z, \varepsilon)=\sum_{n=1}^{\infty} \frac{(-\varepsilon)^{n-1}}{n !} u_{n}(z, \varepsilon), \quad z \in \rho\left(H_{0}\right)
$$

Имеем

$$
\left|\Delta_{0}(z, \varepsilon)\right| \leqslant \sum_{n=1}^{\infty}\left[A_{1}(\varepsilon)\left(\sqrt{\Lambda_{0}}+A_{2}(\varepsilon)\right)\right]^{n}=\frac{A_{1}(\varepsilon)\left(\sqrt{\Lambda_{0}}+A_{2}(\varepsilon)\right)}{1-\varepsilon A_{1}(\varepsilon)\left(\sqrt{\Lambda_{0}}+A_{2}(\varepsilon)\right)}
$$

так как по предположению $\varepsilon A_{1}(\varepsilon)\left(\sqrt{\Lambda_{0}}+A_{2}(\varepsilon)\right)<1$. Однако для детерминанта Фредгольма $\Delta(\lambda, \varepsilon)$ имеет место равенство

$$
\Delta(\lambda, \varepsilon)=1-\varepsilon \Delta_{0}(\lambda, \varepsilon), \quad \lambda \in \rho\left(H_{0}\right) .
$$

Отсюда и в силу неравенства $\varepsilon\left|\Delta_{0}(\lambda, \varepsilon)\right|<1$, справедливого при любых $\lambda \in \rho\left(H_{0}\right)$, получим, что $\Delta(\lambda, \varepsilon) \neq 0$ при всех $\lambda \in \rho\left(H_{0}\right)$. Таким образом, из равенства $(2.5)$ в силу теоремы 2.2 вытекает, что $\sigma_{\text {disc }}(H(\varepsilon))=\varnothing$.

6 Теоретическая и математическая физика, т. 186, № 2, 2016 г. 
ДОкАЗАТЕЛЬСТво ТЕОРЕмЫ 2.3. Пусть $\varepsilon \in(0, a], a \in\left(0, \mu_{0}\right)$ и $a_{0}=b_{0}=a$ в множествах $\Gamma_{k}$ и $\Gamma_{k}^{\prime}$. Имеем

$$
\frac{1}{A_{1}(\varepsilon)\left(\sqrt{\Lambda_{0}}+A_{2}(\varepsilon)\right)}=\frac{D_{1}^{\min } D_{2}^{\min }}{k_{1}^{\max } k_{2}^{\max } \Phi_{1}(\varepsilon)} \frac{1}{D_{2}^{\min } \sqrt{\Lambda_{0}}+\sqrt[4]{\Lambda_{0}^{3}} k_{2}^{\max } \Phi_{1}(\varepsilon)}
$$

Нетрудно проверить, что

$$
\sup _{\varepsilon \in\left(0, \mu_{0}\right)} \Phi_{1}(\varepsilon)=\sup _{\varepsilon \in\left(0, \mu_{0}\right)} \Phi_{2}(\varepsilon)=3
$$

Следовательно,

$$
\gamma(a)=\frac{\varphi_{1}(a) \varphi_{2}(a)}{3 k_{1}^{\max } k_{2}^{\max }\left(2+3 \sqrt[4]{\Lambda_{0}} k_{2}^{\max }\right) \sqrt{\Lambda_{0}}}<\frac{1}{A_{1}(\varepsilon)\left(\sqrt{\Lambda_{0}}+A_{2}(\varepsilon)\right)},
$$

так как $0<D_{2}^{\min }<2$. Таким образом, как легко заметить, при $\varepsilon<\gamma_{0}(a)$ выполняются условия теоремы 2.2 и теоремы 4.1. Следовательно,

$$
\sigma(H(\varepsilon))=\sigma_{\mathrm{ess}}(H(\varepsilon))=\sigma(H(0))=\sigma\left(H_{0}\right)
$$

Тем самым доказана теорема 2.3.

\section{Список литературы}

[1] N. Angelescu, R. A. Minlos, V. A. Zagrebnov, Rev. Math. Phys., 17:10 (2005), 1111-1142.

[2] В. А. Малышев, Р. А. Минлос, Линейные операторы в бесконечночастичных системах, Наука, М., 1994.

[3] Ю. В. Жууков, ТМФ, 107:1 (1996), 75-85.

[4] S. Albeverio, Sh. Alimov, Integral Equations Operator Theory, 55:2 (2006), 153-168.

[5] J. Appell, A. S. Kalitvin, M.Z. Nashed, ZAMM Z. Angew. Math. Mech., 79:10 (1999), $703-713$.

[6] Ю. Х. Эшкабилов, ТМФ, 149:2 (2006), 228-243.

[7] S. Albeverio, S. N. Lakaev, Z. I. Muminov, Russ. J. Math. Phys., 14:4 (2007), 377-387.

[8] Т. Х. Расулов, ТМФ, 163:1 (2010), 34-44.

[9] Ю. Х. Эшкабилов, ТМФ, 164:1 (2010), 78-87.

[10] Ю. Х. Эшкабилов, Р. Р. Кучаров, ТМФ, 170:3 (2012), 409-422.

[11] Ю. Х. Эшкабилов, Матем. тр., 15:2 (2012), 194-203.

[12] Э. Р. Акчурин, Р. А. Минлос, Изв. РАН. Сер. матем., 76:6 (2012), 5-38.

[13] Yu. Kh. Eshkabilov, Cent. Eur. J. Math., 6:1 (2008), 149-157.

[14] Ю. Х. Эшкабилов, Матем. тр., 11:1 (2008), 192-207.

[15] Ю. Х. Эшкабилов, Вестн. НУУз, 2006, № 2, 17-21.

Поступила в редакцию 27.02.2015, после доработки 5.05.2015 